\section{Dor lombar crônica em uma população adulta do Sul do Brasil: prevalência e fatores associados}

\author{
Chronic low back pain in a Southern \\ Brazilian adult population: \\ prevalence and associated factors
}

\author{
1 Programa de Pós-graduação \\ em Epidemiologia, \\ Universidade Federal \\ de Pelotas, Pelotas, Brasil. \\ Correspondência \\ Marcelo Cozzensa da Silva \\ Programa de Pós-graduação \\ em Epidemiologia, \\ Universidade Federal \\ de Pelotas. Av. Duque \\ de Caxias 250, 3o andar, \\ Pelotas, RS 96030-002, Brasil. \\ cozzensa@terra.com.br
}

\begin{abstract}
To identify the prevalence of chronic low back pain (CLBP) and examine factors associated with this condition in a Southern Brazilian adult population, a population-based cross-sectional study was conducted, including 3,182 subjects of both sexes, aged 20 years or over, living in the urban area of Pelotas, Rio Grande do Sul State. The questionnaire included socio-demographic, behavioral, and nutritional variables, as well as characterization of exposure to ergonomic factors in daily activities. CLBP prevalence was 4.2\%. The variables sex, age, marital status, schooling, smoking, body mass index,working in a lying position, heavy physical work, and repetitive movements were associated with CLBP. Prevalence of CLBP is important as it limits normal activities and increases the use of health care services. There may be differences in the ergonomic risk factors for CLBP and low back pain in general.
\end{abstract}

Low Back Pain; Adult Health; Cross-Sectional Studies
Marcelo Cozzensa da Silva 1

Anaclaudia Gastal Fassa 1

Neiva Cristina Jorge Valle 1

\section{Introdução}

As dores lombares atingem níveis epidêmicos na população em geral 1 . Andersson 2 afirma que as lombalgias são comuns na população, sendo que, em países industrializados, sua prevalência é estimada em torno de $70 \%$. Em alguma época da vida, de 70 a $85 \%$ de todas as pessoas sofrerão de dores nas costas 3 . Segundo Teixeira 4 cerca de 10 milhões de brasileiros ficam incapacitados por causa desta morbidade e pelo menos $70 \%$ da população sofrerá um episódio de dor na vida. Nos Estados Unidos, a lombalgia é a causa mais comum de limitação de atividades entre pessoas com menos de 45 anos, é a segunda razão mais freqüente para visitas médicas, a quinta causa de admissão hospitalar e a terceira causa de procedimentos cirúrgicos 5 .

Fazendo parte do contexto de dores lombares, as dores lombares crônicas devem ser tratadas como um problema de saúde pública. Um estudo de base populacional, na Noruega, encontrou prevalências de dor lombar crônica de $2,4 \%$ e $1,7 \%$, respectivamente, para homens e mulheres 6 . Esta morbidade atinge principalmente a população em idade economicamente ativa, podendo ser altamente incapacitante e é uma das mais importantes causas de absenteísmo 7. Este tipo de dor contínua e por longo período de tempo afeta muitos aspectos da vida, podendo levar a distúrbios do sono, depres- 
são, irritabilidade e, em casos extremos, ao suicídio 8 .

A procura por tratamento de dores lombares crônicas aumenta a cada dia. A demanda em hospitais e clínicas ocasiona um aumento no custo de despesas com cuidados com a saúde. O custo de tal demanda é um ônus a mais para os cofres públicos e privados, pois o governo, as indústrias e a sociedade devem arcar com as despesas 9. É grande a quantidade de tempo e recursos gastos com os pacientes portadores deste tipo de morbidade 10.

A dor lombar crônica pode ser causada por doenças inflamatórias, degenerativas, neoplásicas, defeitos congênitos, debilidade muscular, predisposição reumática, sinais de degeneração da coluna ou dos discos intervertebrais e outras 11. Entretanto, freqüentemente a dor lombar crônica não decorre de doenças específicas, mas sim de um conjunto de causas, como por exemplo fatores sócio-demográficos (idade, sexo, renda e escolaridade), comportamentais (fumo e baixa atividade física), exposições ocorridas nas atividades cotidianas (trabalho físico pesado, vibração, posição viciosa, movimentos repetitivos) e outros (obesidade, morbidades psicológicas) 12,13.

Vários estudos epidemiológicos têm descrito o tema dor lombar 8,14,15 mas ainda são poucos os que se referem à dor lombar crônica. Além disso, grande parte dos artigos refere-se a grupos populacionais específicos, tais como o de trabalhadores 16,17, e apresentam heterogeneidades de definição do tema 3,18,19,20. No Brasil foram encontrados poucos estudos sobre o assunto, sendo assim, não há dados de prevalência de dor lombar, ou de dor lombar crônica no país 7,21 .

Este estudo tem como objetivo determinar a prevalência de dor lombar crônica em uma amostra de base populacional de adultos residentes na cidade de Pelotas, Rio Grande do Sul e verificar sua associação com variáveis demográficas, sócio-econômicas, comportamentais, ergonômicas e nutricional.

\section{Metodologia}

Foi utilizado um delineamento transversal para estudar indivíduos de vinte anos ou mais residentes na zona urbana da cidade de Pelotas. A coleta de dados compreendeu o período entre 25 de fevereiro e 10 de maio de 2002, sendo realizada em conjunto por 11 mestrandos do Curso de Pós-Graduação em Epidemiologia da Faculdade de Medicina da Universidade Federal de Pelotas, através da aplicação de um ques- tionário geral criado a partir de questões formuladas pelos referidos mestrandos.

Conforme as informações da contagem populacional da Fundação Instituto Brasileiro de Geografia e Estatística (IBGE) 22, estimou-se a necessidade de visitar aproximadamente 1.600 domicílios, distribuídos em oitenta setores censitários, escolhidos através de amostragem estratificada de acordo com quatro níveis de escolaridade média dos chefes de família de cada setor. A partir dos mapas dos setores e da listagem de todos os domicílios, sorteou-se de forma sistemática as moradias a serem visitadas em cada um deles. Realizou-se a entrevista com todos os moradores com idade igual ou superior a vinte anos, residentes nos domicílios sorteados, excluídas as pessoas incapacitadas para responder o questionário como deficientes mentais e pessoas que tiveram acidente vascular cerebral. Devido à pesquisa ser considerada de risco mínimo solicitou-se aos entrevistados um consentimento informado 23 .

Estimando-se uma prevalência da doença nos não expostos em torno de $3 \%$ e a relação exposto/não exposto variando de 1:1 (sexo) a 1:5 (escolaridade), a amostra estudada $(\mathrm{N}=3.182)$ confere um poder estatístico de $80 \%$ para examinar associações com um risco relativo em torno de 2,0 e um nível de confiança de $95 \%$.

As entrevistas foram realizadas por $33 \mathrm{en}$ trevistadoras as quais tinham, no mínimo, cursado o nível médio de escolaridade. Essas foram selecionadas após o treinamento teóricoprático e a realização de entrevistas supervisionadas no estudo piloto. Cada entrevistadora coletou, em média, dados de quarenta famílias em dois setores censitários. Durante as atividades do trabalho de campo cada mestrando ficou responsável pela supervisão de três entrevistadoras.

As informações foram coletadas através de um questionário pré-codificado com questões fechadas. A codificação foi realizada pelas próprias entrevistadoras logo após as entrevistas e revisadas pelos supervisores do trabalho de campo. Os supervisores também realizaram o controle de qualidade que consistiu na aplicação de questionários com número reduzido de questões a $10 \%$ dos entrevistados.

A presença de dor lombar crônica foi estabelecida através da positividade para dois critérios: um que estabelece a identificação da região lombar como o local da dor em uma figura de pessoa em posição ereta, supina e dorsal com as regiões lombar, torácica e cervical pintadas em cores diferentes 24 , e outro que constata a presença desta dor por sete semanas ou mais ${ }^{3}$. 
Foram analisadas como exposições as variáveis demográficas (cor da pele, sexo, idade e situação conjugal), sócio-econômicas (escolaridade e nível sócio-econômico), comportamentais (baixa atividade física e tabagismo), ergonômicas (trabalho sentado, trabalho em pé, trabalho agachado, trabalho deitado, trabalho ajoelhado, vibração e/ou trepidação, carregar peso, movimento repetitivo) e nutricional (índice de massa corporal - IMC). O nível sócio-econômico foi definido a partir do Critério de Classificação Econômica Brasil que estima o poder de compra das pessoas e famílias urbanas 25. O instrumento utilizado para medir baixa atividade física foi o Questionário Internacional de Atividade Física (IPAQ) versão curta, proposto pela Organização Mundial da Saúde (OMS) e Centers for Disease Control and Prevention (CDC) e a variável tabagismo foi coletada nas categorias de não fumante, ex-fumante ou fumante atual. O IMC dos indivíduos foi medido pelo peso $(\mathrm{Kg})$ referido, dividido pela altura $(\mathrm{cm})$ referida elevada ao quadrado.

As variáveis ergonômicas foram caracterizadas pela percepção do entrevistado identificando entre as opções (nunca, raramente, geralmente, sempre) qual delas caracterizava melhor a freqüência de exposição. Entre as variáveis aferidas estão esforço físico, vibração e repetitividade, bem como posição viciosa caracterizada pela freqüência na qual o entrevistado trabalhava/estudava sentado, em pé, agachado, deitado ou ajoelhado.

O banco de dados foi construído no programa Epi Info 6.0, sendo realizada dupla digitação de cada questionário. Para a análise utilizou-se o programa STATA 7.0.

A análise bivariada examinou tabelas de contingência e a associação estatística foi aferida para valor $\mathrm{p}<0,05$ pelo teste de $\chi^{2}$ de Pearson para heterogeneidade ou tendência linear. A análise multivariada foi realizada através de regressão logística não condicional, que permitiu controle simultâneo de fatores que levaram em conta a hierarquia de determinação da dor lombar crônica. O efeito de delineamento, com valor igual a 1,4 foi considerado na análise.

O modelo proposto para a hierarquia citada foi constituído de três níveis: o primeiro, em que estão inseridas as variáveis demográficas (sexo, idade e cor da pele), o segundo em que estão as variáveis sócio-econômicas (nível sócio-econômico e escolaridade) e demográfica (situação conjugal), e o terceiro que abrange as variáveis ergonômicas (trabalho sentado, trabalho em pé, trabalho agachado, trabalho ajoelhado, trabalho deitado, vibração e/ou trepidação e movimento repetitivo), as variáveis com- portamentais (baixa atividade física, tabagismo) e a variável nutricional (IMC).

Os efeitos das variáveis do primeiro nível foram controlados entre si; as do segundo nível foram controlados entre elas e para as do primeiro nível; as do terceiro nível foram controladas entre elas e para as dos dois níveis anteriores 26. Entraram no modelo hierarquizado de análise todas as variáveis que apresentaram, na análise bivariada, valor $\mathrm{p} \leq 0,2$. As variáveis que, na análise multivariada, também apresentaram valor $\mathrm{p} \leq 0,2$ permaneceram no modelo sempre que preenchiam os critérios para prováveis fatores de confusão. Para seleção das variáveis que permaneceram no modelo de regressão logística foi utilizado o processo de seleção para trás, ficando no modelo final todas variáveis que apresentaram valor $\mathrm{p}<0,05$.

\section{Resultados}

Foram estudados 3.182 indivíduos de vinte anos ou mais em 1.600 domicílios, já descontada a porcentagem final de $5,6 \%$ de perdas e recusas e de $1,1 \%$ de exclusões. A população estudada teve uma média de idade de 44 anos (desvio padrão $=16,3$ anos), sendo que $56,8 \%$ era do sexo feminino e mais de $4 / 5$ da população $(84,7 \%)$ era de cor branca. Quanto à escolaridade, $27,7 \%$ tinha quatro anos ou menos de estudo (média $=7,4$ anos; $\mathrm{dp}=4,3$ anos) (Tabela 1 ).

Dos entrevistados, cerca de $1 / 3$ encontravase no nível sócio-econômico D e E e 61,3\% era casado ou vivia com companheiro. Observou-se que $27,9 \%$ fumava atualmente, $41,1 \%$ era sedentário e $14,3 \%$ apresentava IMC correspondente a obesidade (igual ou superior a $30 \mathrm{~kg} / \mathrm{m}^{2}$ ).

Para definir as prevalências das variáveis ergonômicas, as freqüências obtidas nas categorias "geralmente" e "sempre" foram reunidas em um único grupo. Em relação à posição viciosa observou-se que $35,9 \%$ trabalhava, geralmente ou sempre, sentado; $74,4 \%$, em pé; $5,8 \%$, agachado; $4,5 \%$, deitado e 2,9\%, ajoelhado. Aproximadamente metade das pessoas estudadas realizava movimentos repetitivos; $19,1 \%$ estavam expostos à vibração e/ou trepidação e $24,4 \%$ carregava peso, geralmente ou sempre.

A prevalência de dor lombar crônica na população foi de $4,2 \%$ e, o tempo médio que a dor perdurou nos indivíduos foi de 82,6 dias ( $\mathrm{dp}=$ 14,5 dias).

$\mathrm{Na}$ análise bruta (Tabela 1) observou-se que a dor lombar crônica foi significativamente maior no sexo feminino e, conforme aumentava a idade dos indivíduos, houve uma tendência de aumento linear da freqüência de dor. 
Freqüência simples, prevalência de dor lombar crônica e associação entre variáveis sócio-econômicas e demográficas com dor lombar crônica. Pelotas, Rio Grande do Sul, Brasil, 2002.

\begin{tabular}{|c|c|c|c|c|}
\hline Variáveis & $\mathbf{n}$ & Prevalência (\%) & $\begin{array}{c}\text { Análise bruta } \\
\text { RO (IC95\%) }\end{array}$ & Valor p \\
\hline Sexo $(n=3.182)$ & & & & $<0,001 *$ \\
\hline Masculino & 1.374 & 2,9 & 1,00 & \\
\hline Feminino & 1.808 & 5,2 & $1,88(1,31-2,69)$ & \\
\hline Idade em anos $(n=3.182)$ & & & & $<0,001 * *$ \\
\hline $20-29$ & 719 & 1,0 & 1,00 & \\
\hline $30-39$ & 680 & 3,1 & $3,24(1,42-7,39)$ & \\
\hline $40-49$ & 667 & 5,3 & $5,63(2,47-12,87)$ & \\
\hline $50-59$ & 533 & 7,7 & $8,49(4,06-17,78)$ & \\
\hline $60-69$ & 307 & 4,9 & $5,28(2,26-12,33)$ & \\
\hline 70 ou mais & 276 & 5,3 & $5,70(2,31-14,05)$ & \\
\hline Cor da pele $(n=3.182)$ & & & & $0,7^{*}$ \\
\hline Branca & 2.696 & 4,3 & 1,00 & \\
\hline Não-branca & 486 & 3,9 & $0,92(0,59-1,43)$ & \\
\hline Nível sócio-econômico ( $n=3.170)$ & & & & $0,07^{\star \star}$ \\
\hline Classe A B & 747 & 2,8 & 1,00 & \\
\hline Classe C & 1.270 & 4,6 & $1,62(1,04-2,51)$ & \\
\hline Classe D E & 1.153 & 4,6 & $1,63(0,98-2,71)$ & \\
\hline Situação conjugal ( $n=3.182$ ) & & & & $0,02 *$ \\
\hline Casado ou vive com companheiro & 1.951 & 4,8 & 1,00 & \\
\hline Vive sozinho & 1.231 & 3,2 & $0,65(0,45-0,94)$ & \\
\hline Escolaridade em anos $(n=3.177)$ & & & & $<0,001$ ** \\
\hline 0 & 223 & 6,9 & $3,60(1,59-8,16)$ & \\
\hline $1-4$ & 656 & 6,3 & $3,29(1,70-6,40)$ & \\
\hline $5-8$ & 1.067 & 4,4 & $2,27(1,24-4,14)$ & \\
\hline $9-11$ & 780 & 2,7 & $1,36(0,62-2,94)$ & \\
\hline 12 ou mais & 451 & 2,0 & 1,00 & \\
\hline
\end{tabular}

$\mathrm{RO}=$ Razão de odds; IC95\% = intervalo de confiança.

* Qui-quadrado

** Teste de tendência linear

Essa tendência também foi encontrada na diminuição do nível de escolaridade e do nível sócio-econômico. Na variável situação conjugal, viver sozinho mostrou ser proteção para a dor lombar crônica.

Uma outra observação diz respeito à tendência de aumento linear significativo na prevalência de dor lombar crônica conforme aumenta o IMC, da mesma forma como isso se verifica nos indivíduos que não fumam para os ex-fumantes e fumantes atuais. Também foi observado que a prevalência da dor é maior para quem está exposto ao trabalho deitado geralmente/sempre quando comparado àqueles que nunca o fazem. As variáveis movimento repetitivo e carregar peso não se mostraram as- sociadas ao desfecho, mas foram levadas para a análise multivariada por apresentarem valor $\mathrm{p}<0,2$ (Tabela 2). As variáveis cor da pele, baixa atividade física, trabalho sentado, trabalho em pé, trabalho agachado, trabalho ajoelhado e exposição à vibração e/ou trepidação não se mostraram associadas com a dor lombar crônica na análise bivariada (Tabelas 1 e 2).

Na análise multivariada (Tabela 3 ) as variáveis sexo, idade, situação conjugal, escolaridade, tabagismo, IMC, trabalho deitado, carregamento de peso no trabalho e movimento repetitivo permaneceram significativamente associadas com o desfecho. Já a variável nível sócio-econômico apresentou $\mathrm{p} \leq 0,2$, permanecendo no modelo como fator de confusão. 
Freqüência simples, prevalência de dor lombar crônica e associação entre variáveis comportamentais, ergonômicas e a variável nutricional com a dor lombar crônica. Pelotas, Rio Grande do Sul, Brasil, 2002

\begin{tabular}{|c|c|c|c|c|}
\hline Variáveis & n & Prevalência (\%) & $\begin{array}{l}\text { Análise bruta } \\
\text { RO (IC95\%) }\end{array}$ & Valor $p$ \\
\hline Tabagismo $(n=3.182)$ & & & & $0,03^{\star *}$ \\
\hline Nunca fumou & 1.668 & 3,2 & 1,00 & \\
\hline Ex-fumante & 627 & 5,0 & $1,58(0,92-2,72)$ & \\
\hline Fumante atual & 887 & 5,5 & $1,78(1,15-2,75)$ & \\
\hline $\operatorname{IMC}(n=3.047)$ & & & & $0,01 * \star$ \\
\hline Até 19,9 & 258 & 2,7 & 1,00 & \\
\hline 20 a 24,9 & 1.284 & 3,4 & $1,28(0,58-2,82)$ & \\
\hline 25 a 29,9 & 1.068 & 4,1 & $1,51(0,69-3,30)$ & \\
\hline 30 ou mais & 437 & 6,2 & $2,38(1,03-5,50)$ & \\
\hline Baixa atividade física $(n=3.119)$ & & & & $0,3^{*}$ \\
\hline Não & 1.837 & 3,9 & 1,00 & \\
\hline $\operatorname{Sim}$ & 1.282 & 4,6 & $1,18(0,85-1,65)$ & \\
\hline Trabalho sentado $(n=3.155)$ & & & & $0,8^{* \star}$ \\
\hline Nunca & 433 & 4,2 & 1,00 & \\
\hline Raramente & 1.590 & 4,2 & $1,00(0,53-1,86)$ & \\
\hline Geralmente ou sempre & 1.132 & 4,0 & $0,95(0,52-1,76)$ & \\
\hline Trabalho em pé $(n=3.156)$ & & & & $0,4^{\star \star}$ \\
\hline Nunca & 67 & 7,5 & 1,00 & \\
\hline Raramente & 743 & 4,2 & $0,54(0,21-1,37)$ & \\
\hline Geralmente ou sempre & 2.346 & 4,0 & $0,51(0,20-1,31)$ & \\
\hline Trabalho agachado $(n=3.156)$ & & & & $0,8^{* *}$ \\
\hline Nunca & 1.809 & 4,1 & 1,00 & \\
\hline Raramente & 1.163 & 4,0 & $0,97(0,66-1,41)$ & \\
\hline Geralmente ou sempre & 184 & 4,9 & $1,21(0,62-2,34)$ & \\
\hline Trabalho deitado $(n=3.156)$ & & & & $0,008^{\star *}$ \\
\hline Nunca & 2.556 & 3,7 & 1,00 & \\
\hline Raramente & 456 & 3,5 & $0,94(0,57-1,56)$ & \\
\hline Geralmente ou sempre & 144 & 13,2 & $3,94(1,97-7,87)$ & \\
\hline Trabalho ajoelhado $(n=3.156)$ & & & & $0,4^{\star \star}$ \\
\hline Nunca & 2.331 & 4,3 & 1,00 & \\
\hline Raramente & 734 & 3,5 & $0,82(0,56-1,20)$ & \\
\hline Geralmente ou sempre & 91 & 4,4 & $1,03(0,37-2,83)$ & \\
\hline Vibração e/ou trepidação $(n=3.155)$ & & & & $0,6^{*}$ \\
\hline Não & 2.554 & 4,0 & 1,00 & \\
\hline Sim & 601 & 4,5 & $1,12(0,72-1,75)$ & \\
\hline Carregar peso $(n=3.156)$ & & & & $0,2^{\star \star}$ \\
\hline Nunca & 1.113 & 3,9 & 1,00 & \\
\hline Raramente & 1.272 & 3,8 & $0,98(0,66-1,44)$ & \\
\hline Geralmente ou sempre & 771 & 5,1 & $1,33(0,93-1,89)$ & \\
\hline Movimento repetitivo $(n=3.151)$ & & & & 0,07 * \\
\hline Sim & 1.469 & 3,4 & 1,00 & \\
\hline Não & 1.682 & 4,7 & $1,40(0,97-2,02)$ & \\
\hline
\end{tabular}

$\mathrm{RO}=$ Razão de odds; IC95\% = Intervalo de confiança; IMC = Índice de Massa Corporal.

* Qui-quadrado

** Teste de tendência linear 
Tabela 3

Análise ajustada de fatores associados à dor lombar crônica.

Pelotas, Rio Grande do Sul, Brasil, 2002.

\begin{tabular}{|c|c|c|}
\hline \multirow[t]{2}{*}{ Variáveis } & \multicolumn{2}{|c|}{ Análise ajustada } \\
\hline & RO (IC95\%) & Valor $p$ \\
\hline \multicolumn{3}{|l|}{ 1ㅇNível } \\
\hline $\operatorname{Sexo}(n=3.182)$ & & 0,002 \\
\hline Masculino & 1,00 & \\
\hline Feminino & $1,79(1,24-2,58)$ & \\
\hline Idade em anos $(n=3.182)$ & & $<0,001$ \\
\hline $20-29$ & 1,00 & \\
\hline $30-39$ & $3,24(1,43-7,36)$ & \\
\hline $40-49$ & $5,56(2,44-12,69)$ & \\
\hline $50-59$ & $8,24(3,92-17,30)$ & \\
\hline $60-69$ & $5,12(2,17-12,08)$ & \\
\hline 70 ou mais & $5,39(2,17-13,35)$ & \\
\hline \multicolumn{3}{|l|}{ 2o Nível } \\
\hline Situação conjugal ( $n=3.182$ ) & & 0,004 \\
\hline Casado ou vive com companheiro & 1,00 & \\
\hline Vive sozinho & $0,58(0,40-0,85)$ & \\
\hline Escolaridade em anos $(n=3.177)$ & & 0,01 \\
\hline 0 & $2,26(0,94-5,46)$ & \\
\hline $1-4$ & $2,50(1,23-5,05)$ & \\
\hline $5-8$ & $2,03(1,11-3,72)$ & \\
\hline $9-11$ & $1,46(0,66-3,24)$ & \\
\hline 12 ou mais & 1,00 & \\
\hline \multicolumn{3}{|l|}{ 3o Nível } \\
\hline Tabagismo $(n=3.182)$ & & $<0,001$ \\
\hline Nunca fumou & 1,00 & \\
\hline Ex-fumante & $1,64(0,88-3,05)$ & \\
\hline Fumante atual & $2,36(1,49-3,74)$ & \\
\hline $\mathrm{IMC}(\mathrm{n}=3.047)$ & & 0,04 \\
\hline Até 19,9 & 1,00 & \\
\hline $20-24,9$ & $1,23(0,55-2,77)$ & \\
\hline $25-29,9$ & $1,38(0,61-3,10)$ & \\
\hline 30 ou mais & $2,01(0,86-4,71)$ & \\
\hline Trabalho deitado $(n=3.156)$ & & 0,04 \\
\hline Nunca & 1,00 & \\
\hline Raramente & $0,74(0,46-1,24)$ & \\
\hline Geralmente ou sempre & $2,94(1,61-5,37)$ & \\
\hline Carregar peso $(n=3.156)$ & & 0,04 \\
\hline Nunca & 1,00 & \\
\hline Raramente & $1,09(0,72-1,66)$ & \\
\hline Geralmente ou sempre & $1,67(1,09-2,57)$ & \\
\hline Movimento repetitivo $(n=3.151)$ & & 0,008 \\
\hline Não & 1,00 & \\
\hline Sim & $1,75(1,20-2,55)$ & \\
\hline
\end{tabular}

$\mathrm{RO}=$ Razão de odds; IC95\% = Intervalo de confiança; IMC = Índice de massa corporal. 10 Nível: ajustadas entre elas; 2 o Nível: ajustadas entre elas e para as variáveis do 1ㅇ Nível; 3o Nível: ajustadas entre elas, para as variáveis do 1 o e do 2o Nível e para a variável classe social.
A prevalência de dor lombar, de acordo com a idade, apresentou um aumento linear significativo, sendo que a faixa entre 50 e 59 anos apresenta o maior risco; cerca de oito vezes mais dor lombar crônica do que na faixa entre 20 a 29 anos. A dor lombar crônica aumenta linearmente conforme diminui a escolaridade, sendo que as categorias sem escolaridade, de 1 a 4 e de 5 a 8 anos de estudo tiveram, pelo menos, duas vezes mais risco de apresentar o desfecho do que as pessoas com 12 anos ou mais de escolaridade. Riscos significativos acima de 2,0 também foram encontrados para as pessoas que fumam atualmente quando comparados aos que não fumam, bem como aqueles que exercem suas atividades cotidianas, geralmente ou sempre, deitados quando comparados aos que nunca ficam nesta posição viciosa (Tabela 3).

Dentre os indivíduos que tiveram dor lombar crônica, a maioria $(76,7 \%)$ relatou dificuldade de realizar suas atividades de trabalho. Ainda, 1/4 dos indivíduos que apresentaram esta dor faltou ao trabalho e/ou escola, sendo que $97 \%$ destes faltaram exclusivamente ao trabalho remunerado ou no domicílio. Entre os indivíduos com 40 anos ou mais que apresentaram dor lombar crônica, $27,6 \%$ faltou ao trabalho/escola ou deixou de realizar atividades domésticas por causa da dor. Metade dos indivíduos com esta morbidade procurou algum tipo de assistência, sendo que a procura (não exclusiva) a médico, fisioterapeuta ou massagista foi respectivamente de $90,5 \% ; 27,0 \%$ e $22,2 \%$.

\section{Discussão}

A longa duração e o caráter incapacitante decorrente da dor lombar crônica fazem com que a prevalência encontrada neste estudo seja considerada importante. As variáveis sexo feminino, idade avançada, viver com companheiro, escolaridade baixa, tabagismo, IMC elevado, trabalho deitado, carregar peso no trabalho e realizar movimento repetitivo se comportaram como fator de risco para dor lombar crônica.

Foi estudada uma amostra representativa da população adulta de Pelotas, com um baixo percentual de perdas e recusas $(5,6 \%)$. A descrição das variáveis sócio-demográficas foi condizente com os dados censitários da cidade 22 .

Apesar das vantagens quanto à rapidez e baixo custo, o delineamento transversal apresenta uma limitação inerente que é a possibilidade de causalidade reversa. No caso específico deste estudo, acredita-se que este viés pode afetar as associações do desfecho com as variá- 
veis comportamentais e ergonômicas, mas não com os preditores demográficos e sócio-econômicos.

São poucos os estudos sobre dor lombar crônica, sendo que os existentes avaliam principalmente o tratamento 9,19,27. Assim, os resultados encontrados também serão comparados com os estudos sobre dor lombar em geral que estão em maior quantidade na literatura.

As prevalências de dor lombar crônica em outros estudos de base populacional com critérios para definição de caso semelhantes aos utilizados neste estudo variaram entre $2 \%$ e $6,5 \%$; sendo semelhantes ao encontrado 6,18.

As mulheres apresentaram risco superior ao dos homens para dor lombar crônica. Alguns estudos epidemiológicos atribuem este achado a um viés de informação 28. Porém o achado é plausível, uma vez que as mulheres,cada vez,mais combinam a realização de tarefas domésticas com o trabalho fora de casa onde estão expostas a cargas ergonômicas, principalmente repetitividade, posição viciosa e trabalho em grande velocidade 29. Além disso, o sexo feminino apresenta algumas características anátomo-funcionais (menor estatura, menor massa muscular, menor massa óssea, articulações mais frágeis e menos adaptadas ao esforço físico pesado, maior peso de gordura) que podem colaborar para o surgimento das dores lombares crônicas.

Com o aumento da idade,houve um aumento da prevalência de dor lombar crônica, o que é sustentado por várias pesquisas 1,17,30. A faixa etária de maior risco foi entre 50 e 59 anos,o que está de acordo com a literatura 31 . O risco aumentado nesta faixa de idade pode dever-se ao fato de que os processos degenerativos, de um modo geral, podem estar bem avançados, trazendo como conseqüências o desgaste das estruturas ósteo-musculares e orgânicas 1. Além disso, a maioria das pessoas nesta faixa encontra-se, apesar de próximos da aposentadoria, ainda trabalhando, o que não acontece para a maioria das pessoas acima desta faixa de idade 22 .

Ser casado ou morar com companheiro esteve associado à dor lombar crônica, o que está de acordo com a pesquisa de Lee et al. 15. Provavelmente, situação conjugal não seja um fator de risco para o desfecho, mas, sim, um marcador de risco, podendo estar relacionado, por exemplo, a maiores exposições ergonômicas no trabalho/domicílio ou a características comportamentais de risco.

Consistente com outros estudos, o nível de escolaridade dos indivíduos esteve inversamente associado à prevalência de dor lombar crônica 32. O efeito da baixa escolaridade sobre o desfecho é mediado pela maior exposição a cargas ergonômicas, tanto no domicílio quanto no trabalho, e pelo tabagismo entre outros 33 .

O tabagismo apresentou forte associação com a dor lombar crônica, sendo que algumas teorias têm sido postuladas para explicar tal relação. A primeira está relacionada com a tosse crônica causada pelo hábito de fumar, tendo em vista que a tosse aumenta a pressão interna abdominal e dos discos vertebrais lombares. A segunda se refere ao efeito da nicotina, que produz uma redução da circulação sangüínea e de algumas substâncias como sulfato e oxigênio no corpo vertebral, o que provavelmente reduz a nutrição dos discos vertebrais. Por último, o uso do fumo poderia estar relacionado a fatores psicossociais de risco para o desfecho como baixa condição sócio-econômica, que implicariam em maiores demandas físicas de trabalho, e a estresse 34 .

A prevalência de dor lombar crônica aumenta linearmente com o aumento do IMC, o que está de acordo com alguns estudos 35,36. A “carga extra” que a estrutura osteo-músculoarticular é obrigada a sustentar pode alterar o equilíbrio biomecânico do corpo justificando o risco aumentado de dor lombar crônica em pessoas com sobrepeso e obesidade.

O nível de atividade física não apresentou associação com dor lombar crônica, discordando de alguns estudos que associam indivíduos mais ativos no lazer a índices reduzidos da morbidade 7,37 . No entanto, o instrumento utilizado nesta pesquisa (IPAQ) mede as quatro esferas da atividade física individual (lazer, ocupacional, serviço doméstico e deslocamento). Supõe-se que as atividades de lazer sejam fatores de proteção enquanto as atividades ocupacionais, fatores de risco para as dores lombares crônicas, o que pode ter anulado a associação no presente estudo. Além disso, a falta de associação poderia refletir um viés de causalidade reversa, ou seja, pessoas com dor lombar crônica passaram a ser sedentárias por causa da dor.

Trabalho deitado, carregar peso e realizar movimentos repetitivos foram as exposições ergonômicas que permaneceram associadas ao desfecho no modelo final e estão de acordo com os achados da bibliografia 12,13. Porém, contrariando a bibliografia, outras posições viciosas como trabalho em pé, sentado, agachado ou ajoelhado e vibração/trepidação não se mantiveram no modelo. A ausência de associação entre o desfecho e algumas variáveis ergonômicas pode dever-se a causalidade reversa ou, no caso específico das posições viciosas em 
pé, agachado e ajoelhado, a falta de poder estatístico. Entretanto, este achado pode indicar diferenças nos fatores de risco para dor lombar em geral e dor lombar crônica. Outro aspecto a ser considerado é que as exposições ergonômicas foram medidas não só no trabalho, mas também no domicílio, local em que as cargas ergonômicas podem ter menor intensidade, seja pela sua natureza, seja pela maior autonomia dos indivíduos para não se expor.

Apesar de existir, no município estudado, uma boa rede pública de atenção básica à saúde, bem como de serviços privados, os índices de busca por assistência, entre os indivíduos com dor lombar crônica, encontrados no estudo, ainda são muito baixos quando comparados a países desenvolvidos 5. Dentre os que procuraram assistência, o estudo apontou uma busca a médicos maior do que a encontrada na literatura, porém a busca por outros tipos de profissionais como fisioterapeutas e massagistas foi menor do que a citada por Hansson \& Hansson 9

O absenteísmo relacionado a dor lombar crônica em indivíduos de quarenta anos ou mais foi compatível com o encontrado em um estudo da Noruega 6 e confirmam a importância desta morbidade.

\section{Resumo}

Para determinar a prevalência de dor lombar crônica e examinar fatores associados a esta morbidade em uma população adulta do sul do Brasil foi realizado um estudo transversal de base populacional em 3.182 indivíduos, de ambos os sexos, com vinte anos ou mais, residentes na zona urbana de Pelotas, Rio Grande do Sul. Foram aplicados questionários que incluíam questões sócio-demográficas, comportamentais,nutricionais e exposições a cargas ergonômicas nas atividades cotidianas. A prevalência de dor lombar crônica na população foi de 4,2\%. As variáveis sexo, idade, situação conjugal, escolaridade,tabagismo, índice de massa corporal,trabalho deitado, carregar peso e realizar movimento repetitivo mostraram associação com presença de dor lombar crônica. A prevalência de dor lombar crônica é importante e causa limitação de atividades e procura por serviços de saúde. É possível que existam diferenças nos fatores de risco ergonômicos para dor lombar crônica e dor lombar em geral.

Dor Lombar; Saúde do Adulto; Estudos Transversais
O estudo apontou que a prevalência de dor lombar crônica é importante quando se considera a quantidade de limitação das atividades e de demanda por serviço de saúde que este problema gera. Levantou a hipótese de que existem diferenças nos fatores de risco ergonômicos para dor lombar crônica e dor lombar em geral que podem estar definidas tanto por tipos específicos de exposição quanto por intensidade. Além disso, deixou claro que para examinar a associação entre atividade física e dor lombar crônica é preciso detalhar melhor o tipo e a intensidade da atividade e, preferencialmente, utilizar delineamentos longitudinais.

Cabe ainda ressaltar que o aumento da idade é fator de risco para dor lombar crônica devendo, assim, haver uma redução gradual da exposição a cargas ergonômicas. Além disso, as mulheres apresentam um risco aumentado deste desfecho e devem ter uma carga ergonômica adequada a sua capacidade e peculiaridade física.

Por fim, é importante destacar que os serviços de atenção básica em saúde devem estar preparados para diagnosticar e tratar o problema, bem como identificar suas causas, a fim de serem estabelecidas estratégias de prevenção adequadas a cada caso.

\section{Colaboradores}

M. C. Silva participou na revisão de literatura, elaboração do projeto, elaboração dos instrumentos de coleta de dados, treinamento das entrevistadoras, definição da amostra e logística, coordenação da coleta de dados, análise dos dados, redação do artigo final. A. G. Fassa contribuiu na elaboração do projeto, elaboração dos instrumentos de coleta de dados, treinamento das entrevistadoras, definição da amostra e logística, análise dos dados, redação do artigo final. N. C. J. Valle colaborou na elaboração do projeto, definição da amostra e logística, análise dos dados, redação do artigo final.

\section{Agradecimentos}

Este trabalho contou com o apoio financeiro da Coordenação de Aperfeiçoamento de Pessoal de Nível Superior.

\section{Referências}

1. Deyo R. Low-back pain. Sci Am 1998; 279:48-53.

2. Andersson G. Epidemiologic aspects on low-back pain in industry. Spine 1981; 6:53-60.

3. Andersson G. Epidemiological features of chronic low-back pain. Lancet 1999; 354:581-5. 
4. Teixeira MJ. Tratamento multidisciplinar do doente com dor. In: Carvalho MMMJ, organizador. Dor: um estudo multidisciplinar. São Paulo: Summus Editorial; 1999. p. 77-85.

5. Hart LG, Deyo RA, Cherkin DC. Physician office visits for low back pain: frequency, clinical evaluation, and treatment patterns from a U.S. national survey. Spine 1995; 20:11-9.

6. Hoddevik GH, Selmer R. Chronic low back pain in 40 -year olds in 12 Norwegian conties. Tidsskr Nor Laegeforen 1999; 119:2224-8.

7. Freire M. O efeito do condicionamento físico pela caminhada, na dor lombar crônica [Tese de Doutorado]. São Paulo: Universidade Federal de São Paulo; 2000.

8. Abyholm AS, Hjortahl P. The pain takes hold of life: a qualitative study of how patients with chronic back pain experience and cope with their life situation. Tidsskr Nor Laegeforen 1999; 30:1624-9.

9. Hansson TH, Hansson EK. The effects of common medical interventions of pain, back function, and work resumption in patients with chronic low back pain. Spine 2000; 25:3055-64.

10. Clinical Standars Adivsory Group. Epidemiology review: the epidemiology and cost of back pain. London: Her Majesty's Stationery Office; 1994.

11. World Health Organization. Identification and control of work-related diseases. Geneva: World Health Organization; 1985. (Technical Report Series 714).

12. National Institute for Occupational Safety And Health. Musculoskeletal disorders and workplace factors. 2nd Ed. Cincinnati: National Institute for Occupational Safety And Health; 1998.

13. Marras W. Occupational low back disorder causation and control. Ergonomics 2000; 43:880-902.

14. Valat JP, Goupille P, Rozenberg S, Urbinelli R, Allaert F. Acute low back pain: predictive index of chronicity from a cohort of 2.487 subjects. Spine 2000; 67:456-61.

15. Lee P, Helewa A, Goldsmith CH, Smythe HA, Stitt LW. Low back pain: prevalence and risk factors in an industrial setting. J Rheumatol 2001; 28:346-51.

16. Mendes R. O impacto dos efeitos da ocupação sobre a saúde de trabalhadores. Rev Saúde Pública 1988; 22:311-26.

17. Linton S. Risk factors for neck and back pain in a working population in Sweden. Work Stress 1990; 4:41-9.

18. Frymoyer J. Back pain and sciatica. N Engl J Med 1988; 318:291-300.

19. Hildebrandt J, Pfingsten M, Saur P, Jansen J. Prediction of success from a multidisciplinary treatment program for chronic low back pain. Spine 1997; 22:990-1001.

20. Van Tulder MW, Koes BW, Bouter LM. Conservative treatment of acute and chronic nonspecific low back pain: a systematic review of randomized controlled trials of the most common interventions. Spine 1997; 22:2128-56.

21. Kreling M. Prevalência de dor crônica em adultos trabalhadores [Dissertação de Mestrado]. São Paulo: Universidade de São Paulo; 2000

22. Fundação Instituto Brasileiro de Geografia e Estatística. Censo demográfico 2000. http:/ /www. ibge.gov.br (acessado em 03/Dez/2001).
23. Council for International Organization of Medical Sciences/World Health Organization. International ethical guidelines for biomedical research involving human. Geneva: Council for International Organization of Medical Sciences/ World Health Organization; 1993.

24. Kuorinka I, Jonsson B, Kilbom A, Vinterberg H, Biering-Sorensen F, Andersson G, et al. Standardised Nordic questionnaires for the analysis of musculoskeletal symptoms. Appl Ergon 1987; 18: 233-7.

25. Associação Nacional de Empresas de Pesquisa. Critério de classificação econômica Brasil. São Paulo: Associação Nacional de Empresas de Pesquisa; 1996.

26. Victora CG, Huttly SR, Fuchs SC, Olinto MTA. The role of conceptual frameworks in epidemiological analysis: a hierarchical approach. Int J Epidemiol 1997; 26:224-7.

27. Frymoyer J. Predicting disability from low back pain. Clinical Orthopedic 1992; 279:101-9.

28. Hales TR, Sauter SL, Peterson MR, Fine LJ, PutzAnderson V, Schleifer LR. Musculoskeletal disorders among visual display terminal users in a telecommunications company. Ergonomics 1994; 37:1603-21.

29. Dall'Agnol M. Trabalho e saúde na indústria da alimentação de Pelotas: uma questão de gênero? [Dissertação de Mestrado]. Pelotas: Universidade Federal de Pelotas; 1995.

30. Van Doorn T. Low back disability among self-employed dentists, veterinarians, physicians and physical therapists in the Netherlands. Acta Orthop Scand 1995; 66:1-64.

31. Papageorgiou AC, Croft PR, Ferry S, Jayson MI, Silman AJ. Estimating the prevalence of low back pain in the general population. Evidence from the south Manchester back pain survey. Spine 1995; 20:1889-94.

32. Bergenudd H, Nilsson B. Back pain in middle age; occupational workload and psychologic factors: an epidemiologic survey. Spine 1988; 13:58-60.

33. Vogt M. Prevalência e severidade da dor, cervical e lombar, nos servidores técnico-administrativos da Universidade Federal de Santa Maria [Dissertação de Mestrado]. Florianópolis: Universidade Federal de Santa Catarina; 2000.

34. Boshuizen HC, Verbeek JHAM, Broersen JPJ. Do smokers get more back pain? Spine 1993; 18:35-40.

35. Toda Y, Segal N, Toda T, Morimoto T, Ogawa R. Lean body mass and body fat distribution in participants with chronic low back pain. Arch Intern Med 2000; 160:3265-9.

36. Mortimer M, Wiktorin C. Sports activities, body weight and smoking in relation to low back pain: a population-based case-referent study. Scand J Med Sci Sports 2001;11:178-84.

37. Taimela S, Diederich C, Hubsch M, Heinricy M. The role of physical exercise and inactivity in pain recurrence and absenteeism from work after active outpatient rehabilitation for recurrent or chronic low back pain. Spine 2000; 25:1809-16.

Recebido em 23/Jan/2003

Versão final reapresentada em 14/Ago/2003

Aprovado em 10/Set/2003 